Apidologie, 1985, 16 (1), 39-46

\title{
LEISTUNGSVERGLEICH VON BIENENVOLLKERN SELEKTIERTER UND NICHT SELEKTIERTER HERKUNFT
}

\author{
Hermann PECHHACKER \\ Höhere Bundeslehr- und Versuchsanstalt für Wein- und Obstbau mit Institut für Bienenkunde \\ A - 3400 Klosterneuburg
}

ZUSAMMENFASSUNG

Belegstcllenbegattete Königinnen aus einer selekt:erten Linie waren in Honigleistung und Schwarmträgheit nicht selektierten Königinnen der Landpopulation signifikant überlegen.

Es ist möglich, auch in einer kleinen Population eines Bieneninstitutes einen deutlichen Selektionserfolg zu erzielen. Es ist möglich, gleichzeitig auf höhere Honigleistung und geringere Schwarmneigung zu selektieren.

\section{EINLEITUNG}

Seit 1948 wurde an unserem Institut in Lunz am See Selektion betrieben.

Die vorhandene Zuchtpopulation war anfangs nur auf wenigen Ausgangsvölkern begründet. In den letzten zehn Jahren wurden maximal 500 Völker pro Jahr zur Auslese herangezogen. Die Königinnen dieser Völker wurden größtenteils auf drei institutseigenen Belegstellen und zum geringeren Teil durch künstliche Besamung zur Paarung gebracht.

Wir versuchten, mit einfachen Mitteln die Frage zu klären, wie weit sich durch unsere Zuchtarbeit die Zuchtpopulation gegenüber den nicht züchterisch bearbeiteten Landpopulationen verändert hat. Es wurden die Honigleistung und das Schwarmverhalten in diesem Zusammenhang berücksichtigt. Es schien uns besonders auch die Frage wesentlich, ob es möglich ist, auf erhöhte Honigleistung zu selektieren und dabei zugleich verringerte Schwarmneigung zu erreichen. 


\section{METHODIK}

Auf zwei verschiedenen Standorten (Standort A und Standort B) wurden jeweils vier genetisch verschiedene Geschwistergruppen (entsprechend der Tab. 1) verglichen. Die Königinnen der Linien A und B stammten aus der Zuchtpopulation unseres Institutes. Die Mutter-Königinnen für die Gruppen 3 und 4 wurden zufällig aus einer Imkerei ausgewählt, von der bekannt war, daß noch nie Zuchtmaterial zugekauft und auch keinerlei Auslese durchgeführt wurde. Die Jung-Königinnen der Gruppen 2 und 4 wurden zur Paarung in unmittelbarer Nähe dieser Imkerei aufgestellt.

TAB. 1. - Die auf den Standorten $A$ und $B$ geprïten Abstammungen

TABL. 1. - Different origins tested at bee yards $A$ and $B$

\begin{tabular}{|c|c|c|}
\hline $\begin{array}{l}\text { Gruppe } \\
\text { Group }\end{array}$ & $\begin{array}{l}\text { Mutterkönigin } \\
\text { Breeder queen }\end{array}$ & $\begin{array}{c}\text { Anpaarung } \\
\text { Mating }\end{array}$ \\
\hline 1 & $\begin{array}{l}\text { selektierte } \\
\text { Linie A } \\
\text { selected line } \\
\text { A }\end{array}$ & $\begin{array}{l}\text { selektierte } \\
\text { Linie B } \\
\text { selected line } \\
\text { B }\end{array}$ \\
\hline 2 & $\begin{array}{l}\text { selektierte } \\
\text { Linie A } \\
\text { selected line } \\
\text { A }\end{array}$ & $\begin{array}{l}\text { unkontrolliert } \\
\text { NN } \\
\text { uncontrolled } \\
\text { NN }\end{array}$ \\
\hline 3 & $\begin{array}{l}\text { nicht selektiert } \\
\text { NN } \\
\text { uncontrolled } \\
\text { NN }\end{array}$ & $\begin{array}{l}\text { selektierte } \\
\text { Linie B } \\
\text { selected line } \\
\text { B }\end{array}$ \\
\hline 4 & $\begin{array}{l}\text { nicht selektiert } \\
\text { NN } \\
\text { uncontrolled } \\
\text { NN }\end{array}$ & $\begin{array}{l}\text { unkontrolliert } \\
\text { NN } \\
\text { uncontrolled } \\
\text { NN }\end{array}$ \\
\hline
\end{tabular}

Die Gruppen 3 und 4 stammten von einem Standort in $50 \mathrm{~km}$ Entfernung vom Institut. Es bestanden keine genetischen Beziehungen $\mathrm{zu}$ den Zuchtlinien $\mathrm{A}$ und $\mathrm{B}$.

Auf dem Prüfstandort A wurden je sechs Königinnen jeder Gruppe in Ableger eingeweiselt und mit einer Volksstärke von acht bis neun belagerter Waben eingewintert. Bis auf ein Volk de: Gruppe 2 (dieses Volk wurde über Winter weisellos) konnten alle Völker dieses Standortes in die Auswertung einbezogen werden. Auf dem Standort B wurden sechs Völker der Gruppe 1, drei Völker der Gruppe 2 und drei und fünf Völker der Gruppe 4 ebenfalts in Ableger in der für den Standort A angegebenen Volksstärke eingewintert.

Zur Auswertung gelangten auf diesem Standort sechs Völker der Gruppe 1, zwei Völker der Gruppe 2 und je drei Völker der Gruppen 3 und 4. Ein Volk der Gruppe 2 und zwei Völker der Gruppe 4 gingen über Winter bzw. in der Nachwinterzeit zum Teil durch widrige Umwelteinflüsse (Unruhe durch Mäuse) oder Weisellosigkeit verloren. 
Beim Aufbau der Prüfvölker wurde darauf geachtet, daß alle Völker im Herbst zur Einwinterung die gleiche Stärke aufwiesen (acht bis neun mit Bienen belagerte Waben). Nach der Auswinterung im Frühjahr (4. Mai) wurde die Brutfläche aller Völker vom Standort A gemessen und auf den gleichen Brutstand von ca. 10.250 \pm 550 Brutzellen gebracht. Die Völker des Standortes B wurden nur in der Volksstärke (nach der Zahl der mit Bienen belagerten Waben) ausgeglichen. Die Völker beider Standorte wurden jeweils gleich behandelt (entsprechend der am Institut gebräuchlichen Betriebsweise). Auf dem Standort A wurde kein Absperrgitter, auf dem Standort B aber ein Absperrgitter benutzt. In der verwendeten Betriebsweise wurde keine spezielie Schwarmverhinderung durchgeführt. Die Völker beider Standorte wurden in der ganzen Saison jeweils zweimal durchgesehen, wobei die Honigentnahmen nicht als Durchsichten angesehen wurden.

Neben der Honigleistung wurden das Verhalten und die Schwarmneigung der Völker festgehalten. Dabei wurden Punkte, abgestuft von eins bis vier, vergeben. Ein Punkt $=$ aggressiv bzw. geschwärmt, vier Punkte = auffallend sanftmïtig bzw. schwarmträge. Die Methode der Leistungsprüfung wurde nach der von H. RuTrNer (1972) beschriebenen Methode durchgeführt.

\section{ERGEBNISSE}

Die Ergebnisse der Leistungsprüfung der Stände A und B werden in den Tab. 2 und 3 dargestellt. Die Ergebnisse wurden in Hinblick auf die Honigleistung nach einer zweifaktoriellen Varianzanalyse ohne Wechselwirkung ausgewertet. Die Ertragsunterschiede der beiden Standorte sind signifikant verschieden $(\mathrm{q}=5,57$; $\mathrm{P}<0,01)$.

Die korrigierten Mittelwerte der beiden Standorte betragen bei konstant gehaltener Genetik der Völker $\overline{\mathbf{x}}_{\mathbf{A}}=36,64( \pm 3,01) \mathrm{kg}$ und $\overline{\mathbf{x}}_{\mathbf{B}}=17,11$ $( \pm 3,97) \mathrm{kg}$. Der korrigierte Durchschnittsertrag der genetisch verschiedenen Gruppen betrug bei konstant gehaltenem Standort $\overline{\mathrm{x}}_{\text {Gruppe } 1}=42,05( \pm 4,16) \mathrm{kg}$; $\overline{\mathrm{x}}_{\text {(x ruppe } 2}=23,07( \pm 5,55) \mathrm{kg} ; \overline{\mathrm{x}}_{\text {(7) mppe } 3}=17,33( \pm 4,87) \mathrm{kg}$ und $\overline{\mathrm{x}}_{\text {Gruppe } 4}=$ 25,05 ( \pm 4,87) $\mathrm{kg}$. Nach dem Rangetest nach NEWMAN, KeUls ergibt sich ein statistisch gesicherter Unterschied in der Honigleistung zwischen den Gruppen 1 und 3 ( $\mathbf{P}<0,01$ ) und den Gruppen 1 und 2 bzw. 1 und 4 (jeweils $\mathbf{P}<0,05$ ). Die leistungsmäßige Überlegenheit der Gruppe 1 gegenüber den anderen Gruppen ist unabhängig vom Standort gegeben. Zwischen den Gruppen 2, 3 und 4 ergibt sich jeweils kein statistisch gesicherter Unterschied. Die statistische Auswertung der Schwarmneigung nach dem H-Test nach KRUSKaL und Wallis ergab einen statistisch gesicherten Unterschied zwischen den Gruppen 1 und $4(\mathrm{H}=8,03$; FG $($ Chi 2) $=3 ; \mathrm{P}<0,05)$.

In Bezug auf das Verhalten (Stechlust) ergab sich kein Unterschied zwischen den Gruppen. 
H. PECHHACKER

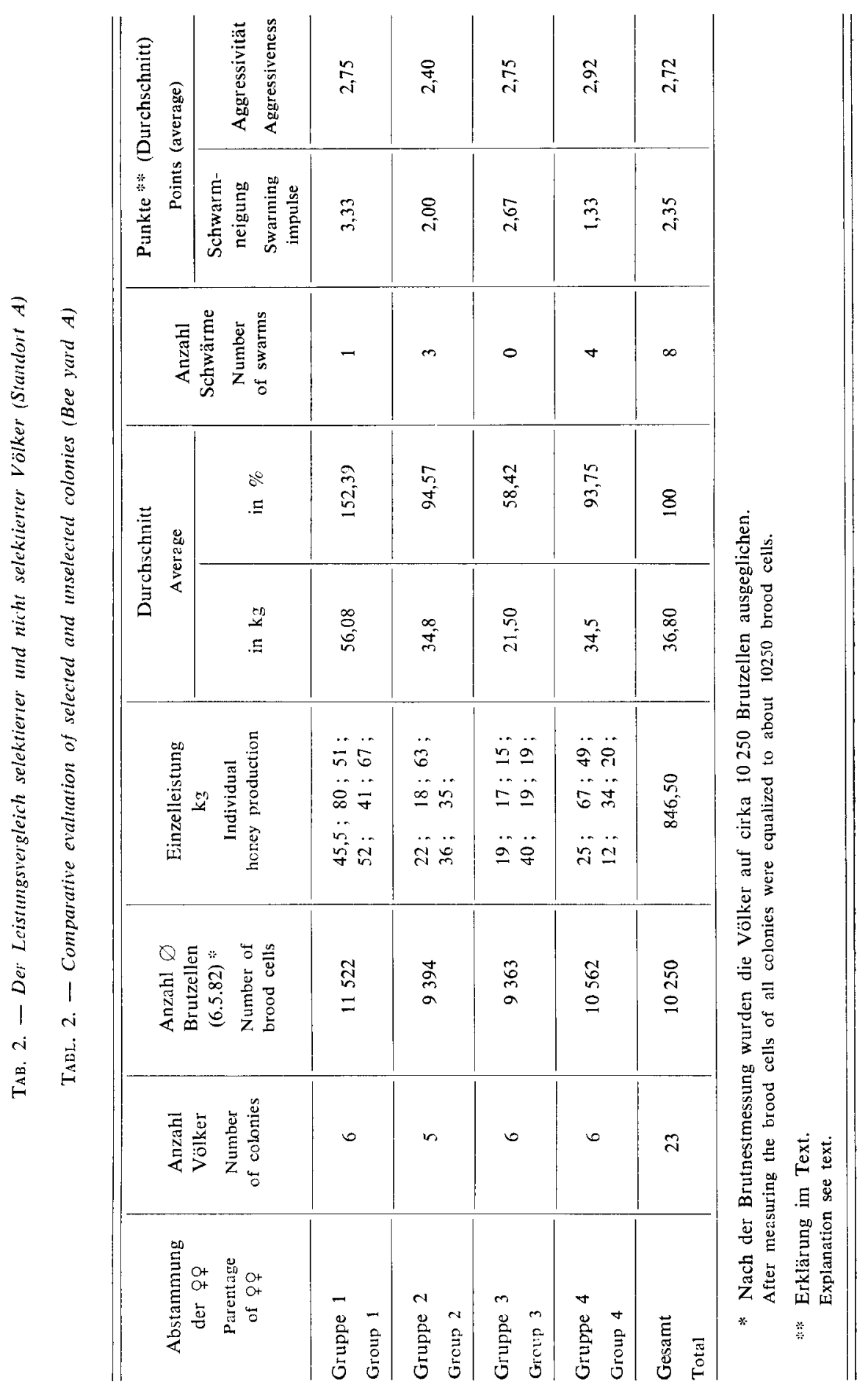


HONIGLEISTUNG VON SELEKTIERTEN LINIEN

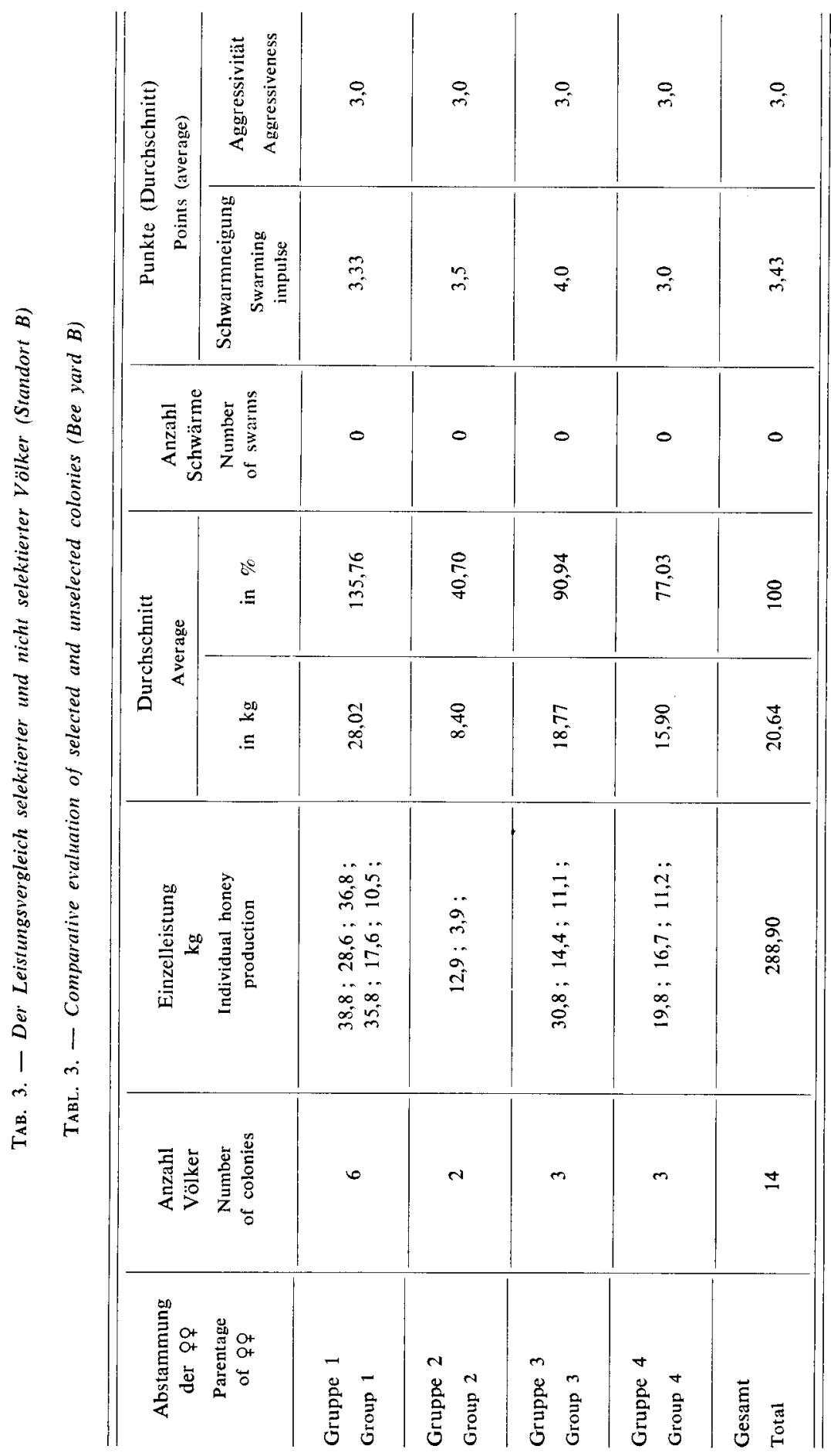




\section{DISKUSSION}

Aus den Ergebnissen ist unabhängig vom Standort eine eindeutige Überlegenheit der Gruppe 1 (selektierte Mutter- und selektierte Vaterlinie) in Bezug auf die Honigleistung zu erkennen. Dies bedeutet, daß die Auslese in der kleinen Zuchpopulation des Institutes einen Erfolg gebracht hat. Hierbei ist nach diesen Ergebnissen Auslese sowohl mütterlicher- als auch väterlicherseits notwendig. Auch die Anpaarung (=Vaterlinie) hat nach diesen Ergebnissen erheblichen Einfluß auf die Honigleistung der Nachkommen (Vergleich Gruppe 1 und 2).

Aus den Paarungskombinationen der Gruppe 3 bzw. Gruppe 2 ist aber feststellbar, daß auch die Kombinationseignung auf Grund der verschiedenen Genome von selektierten und nicht selektierten Herkünften eine wesentliche Rolle spielt.

$\mathrm{Zu}$ ähnlichen Ergebnissen kamen J. FresnaYe et al. (1974) bei der schwarzen Honigbiene (A.m. mellifera L.)

Dies wird bei künftigen Kreuzungen auch innerhalb einer Zuchtpopulation bzw. Rasse zu beachten sein.

TAв. 4. - Leistungsvergleich zwischen Völkern der gleichen Zuchtrichtung wie Tabelle 2 bzw. Tabelle 3 (Zuchtrichtung $A$ ) and zwei anderen, aus der Landpopulation entstandenen und bisher wenig selektierten Zuchtrichtungen $B$ und $C$

TABL. 4. - Comparative evaluation of colonies of line $A$ (same stock as tables 2 and 3) and two other lines ( $B$ and $C$ ) developed from the local population,

hardly ever selected until now

\begin{tabular}{|c|c|c|c|}
\hline $\begin{array}{c}\text { Abstammung } \\
\text { der } \subsetneq \$ \\
\text { Parentage } \\
\text { of } q q\end{array}$ & $\begin{array}{c}\text { Anzahl der geprüften } \\
\text { Völker } \\
\text { Number of tested } \\
\text { colonies }\end{array}$ & $\begin{array}{l}\text { Durchschnittl. Honigprod. } \\
\text { der cinzelnen Gruppen } \\
\text { in \% vom } \\
\text { Gesamtdurchschnitt } \\
\text { Average honey production } \\
\text { of single groups in } \% \\
\text { of total average }\end{array}$ & $\begin{array}{c}\text { Anzahl der Völker } \\
\text { ohne Leistung } \\
\text { Number } \\
\text { of colonies } \\
\text { without honey }\end{array}$ \\
\hline $\begin{array}{l}\text { Zuchtrichtung } \\
\text { Line A }\end{array}$ & 63 & 122,0 & $6(9,5 \%)$ \\
\hline $\begin{array}{l}\text { Zuchtrichtung } \\
\text { Line B }\end{array}$ & 24 & 62,0 & $5(20,8 \%)$ \\
\hline $\begin{array}{l}\text { Zuchtrichtung } \\
\text { Iine C }\end{array}$ & 9 & 51,2 & $2(22,2 \%)$ \\
\hline $\begin{array}{l}\text { Gesamt } \\
\text { Total }\end{array}$ & 96 & 100 & $13(13,5 \%)$ \\
\hline
\end{tabular}

In Tab. 4 werden Leistungsprüfergebnisse einer Großimkerei dargestellt, die ebenfalls einen Vergleich zwischen selektierten Königinnen aus unserer Zucht- 
population mit nicht oder kaum ausgelesenen Herkünften der Landpopulation zulassen.

Wir konnten hier allerdings die Leistung nur in \%-Werten (bezogen auf den jeweiligen Standdurchschnitt) angeben, da uns der betreffende Imker keine Absolutwerte zur Verfügung stellte. Dennoch zeigt sich auch hier die Überlegenheit mütterlicherseits und väterlicherseits selektierter Königinnen gegenüber Königinnen, die nur mütterlichreseits selektiert wurden bzw. von unselektierten Elterntieren abstammen.

In der Literatur sind viele ähnliche Ergebnisse angegeben (F. RUT'INir, 1973 ; V. MaUi, 1978 u.a.). Bei all diesen Leistungsvergleichen erbrachten dic am längsten und konsequentesten selektierten Zuchtlinien den höchsten Ertrag.

Das Verhalten (Aggressivität) der einzelnen Gruppen zeigte keinen Unterschied. Dagegen zeigte das Schwarmverhalten einen signifikanten Unterschied (P<0,05). Die Gruppe 1 mit der höchsten Honigleistung wies mit Abstand die geringste Schwarmneigung auf (durchschnittlich 3,42 Punkte). Die stärkste Schwarmtendenz zeigte die Gruppe 4 (durchschnittlich 1,89 Punkte, von den neun geprüiten Völkern haben fünf geschwärmt, ein Volk konnte nur mit radikalen Maßnahmen am Schwärmen gehindert werden). Diese hohe Schwarmneigung wirkte sich nicht auf die Honigleistung aus, da der Schwarmtrieb erst nach Ende der Tracht (im Juli) einsetzte. Die Königinnen der Gruppe 2 mit nicht selekticrter Anpaarung zeigten ebenfalls cin deutlich schlechteres Schwarmverhalten (die durchschnittliche Schwarmneigung betrug 2,43 Punkte). Hier bleibt die Frage offen, ob der väterliche Einfluß bezüglich der Schwarmneigung größer ist als der mütterliche. Dies kann nur durch weitere Untersuchungen geklärt werden.

Die hier vorliegenden Ergebnisse geben auf eine umstrittene Frage aus der imkerlichen Praxis eine klare Antwort : Man kann durch Selektion eine höhere Honigleistung bei einer gleichzeitig verminderten Schwarmneigung erreichen. Obwohl die Schwarmneigung stark von der Umwelt beeinflußt werden kann, ist aus den vorliegenden Ergebnissen klar zu erkennen, daß auch in Hinblick auf dic Schwarmneigung ein Selektionserfolg erzielt wurde.

In unserer kleinen Zuchtpopulation geschah dies dadurch, daß dic Honigleistung allein als primäres Auslesekriterium gilt und die anderen Kriterien (wie Sanftmut, Schwarmneigung und Rassenreinheit) erst sekundär ausgelesen werden. Das heißt, wenn bei zwei in Bezug auf die Honigleistung gleich veranlagten Völkern eine der Königinnen eine höhere Schwarmträgheit bzw. Sanftmut aufweist, wird dieses Volk für die Nachzucht herangezogen.

In der Leistungsprüfung scheiden sich außerdem in der Regel schwarmfreudige Völker selbst duch verminderte Honigleistung aus der Selektion aus. 


\section{RESUME \\ TEST DE PRODUCTIVITE DE COLONIES D'ABEILLES DOMESTIQUES PROVENANT DE LIGNEES SELECTIONNEES ET NON SELECTIONNEES}

On a comparé la production de miel, le comportement d'essaimage et l'aggressivité de quatre groupes génétiquement différents de colonies d'abeilles, situés dans deux endroits et regroupant 37 ruches au total (Tabl. 1).

La production de miel dans les deux endroits est significativement différente (Tabl. 2 et 3 ). Le groupe qui provient d'une reine sélectionnée et fécondée par des mâles d'une lignée sélectionnée venant de la même population a montré une nette supériorité dans la production de miel, et ceci pour les deux localisations (Tabl. 2 et 3). On n’a pas trouvé de différences entre les groupes en ce qui concerne le comportement (agressivité). Le groupe ayant des parents sélectionnés a montré une tendance significativement plus faible à essaimer que le groupe ayant des parents non sélectionnés.

Il est donc possible d'effectuer avec succès une sélection même à l'intérieur d'une petite population. On peut réussir à sélectionner à la fois les deux caractères suivants : production de miel plus forte, tendance à l'essaimage plus faible.

\section{SUMMARY \\ PRODUCTIVITY TEST OF HONEY BEE COLONIES FROM SELECTED AND NON SELECTED DESCENT} lation.

This paper gives the results of comparison between a selected and an unselected bee popu-

Four genetically different groups of bee colonies (table 1) in two different locations, comprising a total of 37 hives, were compared in regard to honey yield, swarming behaviour and aggressiveness.

Honey production at the two locations was significantly different (table 2 and 3). In both locations clear superiority in honey production was shown in the group originating from a selected breeder queen mated with drones of a selected line from the same population compared to the other groups (table 2 and 3). No difference between groups was found in behavior (aggressiveness). The group with selected parents showed significantly less inclination to swarm than the group with non selected parents.

Thus it is possible to achieve an obvious success in selection even in a small population. Successful selection for higher honey production and lower swarming tendency can be achieved at the same time.

\section{LITERATUR}

Fresnaye J., Lavie P., Boesiger E., 1974. - La variabilité de la production du micl chez l'abeille de race noire (Apis mellifica L.) et chez quelques hybrides interraciaux. Apidologie, 5 (1), 1-20.

MaUL V., 1978. - Leistungsprüfung mit Buckfast- und Carnicamaterial. Allg. dtsch. Imkerztg., $1978(5), 140-142$.

RUtTNER F., 1973. - Zuchttechnik und Zuchtauslese bei der Biene. 3. Aufl. Ehrenwith-Verlag. München, $138 \mathrm{p}$.

RuttNer H., 1972. - Paarungskontrolle und Selektion bei der Honigbiene. Intern. Symposium, Lunz am See, Österreich. Apimondia-Verlag, Bukarest. 